\title{
Die Zukunft der Derivation oder: Derivation 2.0
}

\author{
Nanna Fuhrhop (Oldenburg) und Martina Werner (Wien)
}

\begin{abstract}
Traditionally, contemporary German is considered to be rich in affixes which is displayed by a wide range of e. g. nominal suffixes (such as -ung, -heit, -nis, -tum, -sal). However, productivity tests, especially with non-native lexemes, challenge this view since many formal restrictions between affixes and different word classes can be formally identified - synchronically and diachronically - and which cannot be explained by traditional approaches. This paper questions the general morphological productivity of derivation coinciding with a decrease of nominal, adjectival and verbal affixation and, in parallel, pointing to morphological alternatives. In this view, a process of an increasing "syntactification" (as it will be called) is taking place resulting in a morphological preference for conversion. Diachronically, the morphological development from compounding to derivation is well-described. The question as to why and how conversion emerges, especially in an inflectional language, and how it is linked to former or coexisting morphological types, here derivation, has never been asked - though important observations from language typology have been made. Against this background, the process of syntactification fills this research lacuna, also in a morpho-theoretical way, since it can be interpreted as an ongoing language change consisting of a change in linguistic encoding.
\end{abstract}

\section{$1 \quad$ Einleitung: Zum Status der Wortbildung}

\subsection{Wortbildung als Syntaktisierung}

Im Folgenden geht es - etwas provokant - um die Frage, ob die Ableitung, insbesondere die substantivische Ableitung, wirklich so beständig ist wie in der Forschung suggeriert, oder ob im Gegenteil die Wortbildung im Sinne einer Syntaktisierung durch Wort-,Bildung“ in der Syntax abgelöst wird. Dieser Aufsatz soll einige Aspekte, die in den letzten Jahren in der Forschung betrachtet wurden, zusammenbringen und im Zusammenhang neu interpretieren. Basierend auf bisherigen Erkenntnissen zur Diachronie des Wortbildungstyps Derivation, der neben der Komposition bekanntlich einer der Hauptwortbildungstypen des Deutschen darstellt, soll im Folgenden die Frage einer künftigen Entwicklung im Vordergrund stehen. Da es sich bei den im Folgenden skizzierten Entwicklungslinien um aller Wahrscheinlichkeit mögliche, nicht aber notwendige Tendenzen handelt, kommt dem vorliegenden Beitrag prognostischer Charakter zu. Da Prognosen nur vor dem Hintergrund vergangener Entwicklungen faktenbasiert und im Rahmen der Beobachtungen als mögliche Wahrscheinlichkeiten (im Gegensatz zur Teleologie mit Absolutheitsanspruch) aufgestellt werden können, gelten sie wissenschaftstheoretisch als legitim (i. S. v. lege artis) im Rahmen wissenschaftlicher Forschung. 
In der Wortbildung geht es traditionell einerseits um den bestehenden Wortschatz und seine interne Strukturiertheit und andererseits um Neubildungen (traditionell als „Erweiterung des Wortschatzes" gefasst). Bei den Neubildungen geht es parallel dazu um das Potential einzelner Wortbildungsmuster, d. h. um die Produktivität. In diese doch sehr unterschiedliche Betrachtung kann man wohl heutzutage die Diskussion der Vergangenheit einordnen: In den 1980er Jahren wurde über die Ähnlichkeit (oder Gleichheit) von Syntax und Wortbildung diskutiert (cf. Höhle 1982; Reis 1983); hier ging es wesentlich um produktive Affixe. In den 1990er Jahren entstand die Sicht der paradigmatischen Morphologie; es ging um Vorbilder - der bestehende Wortschatz ist damit wieder in das Sichtfeld geraten (z. B. Becker 1990). Inzwischen ist eine Verbindung beider Sichtweisen zumindest teilweise konsensfähig. Aus Analogien können Muster abgeleitet werden, die dann produktiv werden. Dabei kann es über Affixe zu grammatisch geformten Wort-Bildungen über bestimmte Muster kommen - so wie in der Flexion. Damit ist grundsätzlich die Möglichkeit gegeben, zwischen besonders starken (quantitativ, reihenbildend) und eher schwachen Analogien zu unterscheiden oder mit Fleischer (1976) „Produktivität” und „Aktivität” anzunehmen. Diese Unterscheidungen werden relevant, wenn es um einzelne Neubildungen geht. Wie wäre es zu bewerten, wenn man in einem großen Korpus einen Beleg von Cancel(l)ung findet? Einzelne Wortbildungen sind u. E. allein schon durch starke Vorbilder möglich; durch einzelne Wortbildungen wird ein Suffix also nicht „produktiv”. Außerdem ist, um bei dem Beispiel canceln - Cancellung zu bleiben, zu beachten, dass offenbar der substantivische Infinitiv das Canceln sehr viel üblicher ist und auch den befragten Sprecher/innen des Deutschen als natürlicher erscheint.

Basierend auf diesen Grundüberlegungen kommen wir zu unserer Gesamtthese: Die Suffixe verschwinden zunehmend aus der produktiven Wortbildung zugunsten nicht explizit durch ein Formmerkmal gekennzeichnete Formen; die Wortbildung wird zunehmend im Sinne einer Syntaktisierung realisiert; hierbei ist der Übergang von einer ,(lexikalisch-)morphologischen“ zu einer „morphosyntaktischen“ Wortbildung gemeint. Der Titel „Derivation 2.02 meint vor dem Hintergrund ihrer Beschreibung den Übergang von einem statischen zu einem dynamischen System, ganz wie „2.0“ auch in der Informationstechnologie genutzt wird (cf. O’Reilly 2007: 17).

Vor dem Hintergrund der jeweiligen Wortarten werden zunächst die substantivischen Suffixe im Hinblick auf die Ermittlung ihrer jeweiligen Produktivität fokussiert. Anschließend wird überprüft, ob durch substantivische Suffixoide „Neuzugänge“ bei den substantivischen Suffixen zu erwarten sind. Anschließend folgt die analoge Betrachtung der adjektivischen Suffixe und Suffixoide. Verbale Suffixe spielen aufgrund mangelnder Produktivität im Gegenwartsdeutschen nur am Rande eine Rolle und werden dementsprechend kurz abgehandelt. Präfigierung und Partikelverbbildung werden im vorliegenden Beitrag nicht betrachtet.

\subsection{Axiomatik}

Die Produktivität von Suffixen ist bekanntlich vielfältig diskutiert und es ist schwer, einen allgemein anerkannten Produktivitätsbegriff zu finden. Die Produktivitätskriterien unterteilen sich zunächst in quantitativ und qualitativ. Das Spektrum geht von Hapax legomena (quantitativ, basiert auf großen Korpora, z. B. Baayen/Renouf 1996) bis hin zu einer Intuition von Einzelsprechern. 
Grundidee des vorliegenden Artikels ist, dass das System der Wortbildung genauso wie die Syntax Distributionen bzw. Restriktionsprinzipien unterworfen ist, welche mithilfe von Produktivitätstest ermittelbar sind. Damit sind systematisch auftretende, morphologische Restriktionen (und deren deskriptive Generalisierungen) für die Wortbildung(stheorie) genauso ernst zu nehmen wie syntaktische Restriktionen für die Syntax. Umgekehrt ergibt sich aus dieser Überlegung, dass Wortbildungsprodukte, wie sie z. B. in Korpora zu finden sind, zwar ein Abbild für die Tilgung (ehemaliger) lexikalischer Lücken sind, andererseits jedoch stets auch ein (ggf. beschränktes) Abbild morphologischer Dynamik widerspiegeln, da nicht zwischen produktiven vs. ererbten Bildungen differenziert werden kann. Damit stehen produktive neben lexikalisierten Wortbildungsprodukten, deren jeweiliger Einzelbeispielstatus bei der Widerlegung systematischer Wortbildungssystematizitäten nicht geeignet ist, weil Einzelbeispiele per definitionem nicht reihenbildend ( $>$ Systemgedanke auf quantitativer Ebene) und zunächst ihrerseits in Bezug auf ihre Repräsentativität (> Systemgedanke auf qualitativer Ebene) zu prüfen wären. Somit sind ausschließlich synchrone Distributionsverfahren und die sich daraus ergebenden Restriktionen, wie sie im folgenden Artikel als Ausgangspunkt für deskriptive Generalisierungen verwendet werden, das Mittel der Wahl zur Bestimmung synchroner Produktivität bzw. der Trennung zwischen synchron produktiven vs. synchron nicht-produktiven, ererbten Bildungen.

Morphologietheoretisch kann man mit Distributionstests nachweisen, dass einige Suffixe wie beispielsweise -sam nicht mehr produktiv sind, insbesondere, wenn diese mit entlehnten Basen kombiniert werden (cf. Aronoff 1976), cf.: *surfsam, *chatsam, *mailsam. Vor diesem axiomatischen Hintergrund wird in Abschnitt 1 diskutiert, welche Suffixe als produktiv gelten bzw. inwieweit sich hierfür aus der formalen Substanz zu gewinnende Argumente finden lassen. Bei den Suffixen prüfen wir erstens, ob sie historisch eingeschränkt (das diachrone Kriterium für nachlassende Produktivität) oder erweitert werden (zunehmende Produktivität im Hinblick auf die diachrone Dynamik der Derivationsbasen cf. z. B. Scherer 2005 zum Suffix -er).

Auf Basis der genannten Kriterien wollen wir im Folgenden die einzelnen lexikalischen Wortarten einer eingehenden Prüfung in Hinblick auf die Produktivität ihrer jeweiligen Derivationsmuster unterziehen. In Abschnitt 2 soll zuerst das Substantiv, in Abschnitt 3 das Adjektiv, in Abschnitt 4 das Adverb und in Abschnitt 5 das Verb betrachtet werden.

\section{Das Substantiv}

\subsection{Substantivische Suffigierung}

Von den substantivischen Suffixen werden noch die folgenden als produktiv im Sinne von restriktionslos reihenbildend angesehen (in Anlehnung an Draeger 1996): zur Verbnominalisierung die Suffixe -ung, -erei, Ge-...-e zur Adjektivnominalisierung die Suffixe -heit/-keit/-igkeit, zur Bildung von Individuativa das Suffix -er sowie aus dem Bereich der Modifikation die Morpheme -chen/-lein sowie -in. Nach einer jeweiligen Diskussion der Suffixe und der Berücksichtigung synchroner Sprachdynamik sollen im Anschluss daran die Ergebnisse zusammengeführt werden.

-ung ist nach Demske (2000) und Werner (2010, 2012) zunehmend unproduktiv geworden. Wörter wie *Ansuchung, Zusagung, Verkauffung, Visitirung, Spolirung (cf. Demske 2000: 373) 
sind heutzutage ungrammatisch. Noch im Frnhd. war es nach Demske (2000) möglich, von nahezu allen Verben eine -ung-Ableitung zu bilden. Die Reihenbildung in Bezug auf die Ableitungsbasen und damit die Produktivität von -ung wurden somit zunehmend reduziert. ${ }^{1}$ Im heutigen Deutsch gibt es keineswegs -ung-Ableitungen von allen Verben, sondern wesentlich von terminativen Verben wie Befürchtung - *Fürchtung, Befahrung, Umfahrung - *Fahrung. In dieses Muster würde auf lange Sicht aber ?Screenung, ?Cancellung, ?Coachung, ?Dumpung (Lohndumpung), ?Kidnappung, ?Liftung, ?Likung hineinfallen. Alle diese Bildungen sind zumindest ungewöhnlich. Stattdessen finden sich hier zum Teil auch im Deutschen Bildungen mit -ing wie Coaching, Dumping (Lohndumping) und Screening; insgesamt üblicher scheint aber auch der substantivische Infinitiv wie Liken, Canceln statt Liking, Cancelling.

Der substantivische Infinitiv wird laut Kloocke (1974) im Mittelhochdeutschen produktiv. Auch bei Entlehnungen wie das Chatten, das Surfen, das Chillen ist dieses Muster produktiv. Die These ist also, dass der substantivische Infinitiv - also die syntaktische Umkategorisierung - das zur Kodierung von Verbalabstrakta produktive Wortbildungssuffix -ung in der Produktivität verdrängt hat. -ung ist zwar sehr präsent im Wortschatz des heutigen Deutsch, aber eben in dem beschriebenen Sinne nicht mehr produktiv.

Neben -erei wird häufig auch das Zirkumfix Ge-...e gestellt, auch aufgrund der pejorativen Konnotation beider Bildungsmuster. Mit beiden Bildungsmustern können im heutigen Deutsch neue Wörter gebildet werden, aber sie sind aufgrund ihrer pejorativen Konnotation auf bestimmte Kontexte festgelegt (die Chatterei (3 Belege in W-öffentlich²), die Twitterei (55), die Likerei (0) - das Gechatte (6), das Getwitter (40), das Gelike (0), während diese pragmamorphologische Einschränkung weder für -ung, noch für den substantivischen Infinitiv zutrifft. Die Zirkumfigierung gilt zwar als mit Simplizia und Partikelverben (wie Gelaufe, Hineingelaufe), jedoch nicht mit Präfixverben kombinierbar (*Vergestehe/*Geverstehe). Bei näherer Betrachtung zeigt sich zudem, dass sich die Derivationsmöglichkeit hier eigentlich nur auf die verbale Basis, nicht auf die gesamte Partikelverbbildung bezieht, da das Zirkumfix zwischen, nicht vor Verbstamm und Partikel tritt (cf. Hinein-ge-lauf-e/*Ge-hinein-lauf-e). Somit sind also ausschließlich verbale Simplicia über die Zirkumfigierung nominalisierbar. Mit der unter 1.2 getroffenen Aussage, gemäß der ein Wortbildungstyp eine Wortart in ihrer Gesamtheit (domainspecific word formation nach Gaeta/Ricca 2015), d. h. restriktionslos, erfassen muss, ist die Zirkumfigierung nicht kompatibel, weshalb sie als nicht in vollem Umfang produktiv eingestuft werden muss. Beim Suffix -erei hingegen lässt sich eine entsprechende Basenbeschränkung nicht finden. In beiden Fällen jedoch steht als pragmatisch neutrales Bildungsmuster seit der

\footnotetext{
${ }^{1}$ Schneider-Wiejowski (2011) kommt hier zu einem anderen Ergebnis. Sie berechnet, dass -ung produktiv sein müsse, die Type-Token-Relation nach Baayen sowie andere Werte seien bei -ung konstant hoch, ebenso die Hapaxe usw. Interessant ist, dass bei all ihren Zahlen 1925 fast genauso hoch liegt wie 1999 und dazwischen stets ein „Einbruch” zu verzeichnen ist. Fakt ist, dass es sehr viele -ung-Bildungen im Deutschen gibt, die weitgehend durchsichtig sind und sich deswegen auch „halten” können in dem Sinne, dass sie sofort analysierbar und verständlich sind. Wenn man dies alles mitbedenkt, folgen wir eher der Analyse von Demske: Die Basen sind strukturell beschränkter als noch im Frühneuhochdeutschen. Schneider-Wiejowski zitiert Demske (2000) an verschiedenen Stellen, dennoch verfolgt sie einen quantitativen Produktivitätsbegriff und kommt zu einem anderen Ergebnis, was allerdings auch damit zu tun hat, dass der betrachtete Zeitabschnitt sehr viel kleiner ist.

2 W-öffentlich in Cosmas II, Zugriff am 08.07.2015.
}

ISSN 1615-3014 
zunehmenden Inproduktivwerdung von -ung zur Nominalisierung von Verben der substantivierte Infinitiv bereit.

-heit/-keit ist im Gegenwartsdeutschen für Nominalisierungen von Adjektiven und Partizipien (noch) produktiv, so, v. a. im mündlichen Diskurs, auch für Entlehnungen (wie Coolheit, Gechilltheit, cf. Aronoff 1976). Während das Suffix -heit Nominalisierungen zu suffixlosen Adjektiven und Partizipien bildet, wählt -keit suffigierte Basen. Die Variante -igkeit (ebenfalls für einfache Basen wie Schnell-igkeit) ist heute in dieser Funktion nicht mehr produktiv (*Cooligkeit, *Hippigkeit) und vor dem Hintergrund seiner diachronen Entwicklungsgeschichte stark eingeschränkt produktiv, da es lediglich bei depersonalen Adjektivierungen als Nominalisierungsmittel in Gebrauch ist (wie in Studentenhaftigkeit, Professorenhaftigkeit).

Wie bereits an den deverbalen Nominalisierungen zu sehen war, liegt gerade in der verbalen Basis der Partizipien die Möglichkeit eines Kodierungswechsels: So ist es im Gegenwartsdeutschen möglich, anstelle von Verliebt-heit, Verlegen-heit, Abgeschlagen-heit die jeweiligen substantivische Infinitive mit -sein wie Verliebt-sein, Verlegen-sein, Abgeschlagen-sein zu verwenden. Auch semantisch ist, wie Bücking (2012) $)^{3}$ gezeigt hat, eine solche Verschiebung (es handelt sich in beiden Fällen um Zustände/States) möglich wie sein Beispiel Müdesein zeigt, das bereits ein (primäres) Adjektiv enthält und die Prozesslesart aufruft, ebenso Fremdsein vs. Fremdheit. Einige -heit-Ableitungen sind blockiert wie zum Beispiel ?Altheit wegen Alter. Interessant ist aber, dass Altsein offenbar heutzutage nicht durch Alter blockiert wird. Auch mit aus dem Englischen entlehnten Basen scheint eine Verbindung mit sein gut möglich, die mit -heit ist beschränkt: high - ??Highheit-Highsein, stoned - ??Stonedheit-Stonedsein, hip - ??Hipheit - Hipsein (jeweils mit der Möglichkeit, die sein-Infinitive mit Bindestrich zu schreiben).

Die Argumentation ist hier also, dass zwar ein produktives Suffix vorliegt, es sich aber Ersetzungstendenzen zeigen, die diachron zunehmen, und zwar über die Perfektpartizipien hin zu den genuinen Adjektiven (cf. Werner 2010). Das Einfallstor im Sinne einer Systemlücke für den Abbau ist möglicherweise genau hier zu vermuten. Inwieweit -heit ggf. schon erkennbar an Produktivität verliert, wäre hier eine interessante Forschungsfrage (zur Frage der quantitativen Produktivität cf. Schneider-Wiejowski 2011).

-er ist im heutigen Deutschen produktiv. Es hat sich auch immer weiter ausgeweitet, was durchaus als Hinweis auf Produktivität gesehen wird (z. B. Nübling 2013). Die Ausbreitung geschieht ausgehend von nominalen Basen (belegt sind meist nomina agentis wie Gärtner) hin zu verbalen Basen (belegt sind nomina instrumenti Bohrer sowie nomina acti wie Seufzer). Auch wird parallel dazu eine substantivische Basis konstatiert (Benzin-der Benziner, cf. Scott 2009).

Den Nomina agentis droht allerdings Konkurrenz durch das substantivische Partizip I (Studierende, Lehrende, Argumentierende). Diese Bildungen sind sicher in ihrer Ausbreitung durch

\footnotetext{
${ }^{3}$ Bücking (2012) stellt eine Parallelisierung zwischen -heit- und -ung-Nominalisierungen auf und diskutiert in diesem Zusammenhang synchrone Unterschiede der Semantik der Bildungsmuster Müdigkeit und Müde-Sein. Da der vorliegende Beitrag formale Distributionsverschiebungen unter dem Aspekt sprachlicher Dynamik in den Blick nimmt und damit semantische Unterschiede nicht ausgeschlossen sind, sind die Ergebnisse von Bücking auf semantischer Ebene nicht nur parallel zur Diachronie der Nominalisierungsmuster von -ung vs. dem substantivischen Infinitiv, sondern stützen im Gegenteil die hier getroffenen Aussagen.
} 
sprachnormative Einflüsse wegen der Unterspezifiziertheit hinsichtlich der Semantik des natürlichen Geschlechts in den Pluralformen gefördert worden, was weitreichende Konsequenzen für den natürlichen Sprachgebrauch hatte. So finden Bülow/Herz (2014) bei der Untersuchung der Straßenverkehrsordnung eine Reihe von Singularformen, die die ursprüngliche Intention der Gendergerechtigkeit ad absurdum führen, wie z. B. die Singularformen als Veranstaltender, dem Fahrzeugführenden oder mit dem oder der Verständigen usw. (cf. Bülow/Herz 2014: 502). Die ursprüngliche, sprachnormative Intention ist verschwunden; dass sich entsprechende Formen dennoch so verbreiten, spricht für die Syntaktisierung der Wortbildung.

Für diese These lassen sich weitere Beobachtungen aus der Literatur anführen: So finden Fleischer/Barz (2012: 201f.) konkurrierende Formen wie Alleinerziehende und nur im österreichischen Standard auch Alleinerzieher. Ein möglicher Bedeutungsunterschied ist zwar manchmal vorhanden wie in Denkende - Denker, Schiebende - Schieber (-er-Personenbezeichnungen mit festen Merkmalen, ebd.), aber nicht durchgängig, zumal bei Vorsitzende, Reisende, Streikende die entsprechenden -er-Formen fehlen und sie wohl nur in der Partizip-I-Version vorkommen. Vielreiser kommt im Netz vor; Vielreisende sehr viel häufiger.

Das er-Muster wird durch Entlehnungen aus dem Englischen genährt wie Jogger, Blogger, Chatter, Checker, Dater, Dealer usw.; auch die Nomina instrumenti (Typ: Bohrer, Schleifer, Sauger) werden zusätzlich aus dem Englischen (mit-)motiviert wie Scanner, Beeper, Decoder, DVD-Player. Man findet zudem auch Neubildungen wie Bringer oder Knaller (wie in Das ist der Bringer/Knaller). Es bleibt aber in jedem Fall eine offene Frage, ob es auch englische Verben zur Basis nimmt, wenn die -er-Ableitung nicht aus dem Englischen mitübernommen wird. Zumindest jedoch hinsichtlich des zugewiesenen Genus der Bildungen ist von einer formalen Analogie zum Genus der deutschen -er-Nominalisierungen auszugehen (cf. Chan 2005); auch die Flexionsklassenzuordnung spricht dafür (der Beeper - die Beeper).

Die sog. Nomina acti (wie Ausrutscher, Seufzer, Hopser, Stöhner), also semelfaktive Nominalisierungen (cf. Schäfer 2012), sind das historisch jüngste Muster. Sie sind nicht zu allen Verben bildbar, denn häufig sind die Bildungen blockiert: *Kucker (dafür Blick), *Überschwemmer (wegen Flutwelle), *Anfasser (wegen Berührung), *Anheizer (wegen Hitzeschub). Das Muster ist erst seit dem Neuhochdeutschen produktiv (cf. die Daten in Moser 1993), das heißt nach wie vor liegen Restriktionen vor, weshalb eine generelle Expansion des Musters synchron (ggf. noch) nicht zu dokumentieren, aber künftig nicht auszuschließen ist.

Es finden sich in lexikalisierten Einzelfällen auch andere konvertierte Adjektive, die auf Verben zurückzuführen sind wie der Hoffnungsvolle statt ?der Hoffer, wohl in Analogie zu der Einsichtige (?der Einsehende; ?der Einseher), der Widerspenstige (ohne formales Korrelat), usw. Unter dem Aspekt der Bereitstellung von Nomina agentis für das Sprachsystem lassen sich jedoch noch weitere deskriptive Generalisierungen aus formalen, suffixalen Distributionsbeschränkungen ableiten: Wenn Partizip I die Agens-Rolle übernimmt, so übernimmt das Partizip II die Patiensrolle (cf. Rapp 1996), daher ist an dieser Stelle auch nach den substantivischen Part. II Ausschau zu halten. Denn das Suffix -ling, das gemeinhin im Ruf steht, diese Funktion zu übernehmen, ist nicht mehr produktiv: *Antreffling, *Haarschneidling, *Betrachtling, *Anchatling, so wie Prüfer - Prüfling ,x, der ge-x-t wird; jmd., der zu prüfen ist'. Tatsächlich machen die substantivischen Partizipien II ebenfalls einen Großteil der Substantivierungen aus: der Gelehrte, der Angefeindete, der Behinderte, der Verunglimpfte, auch hier in Analogie zu 
substantivischen Adjektiven. Man könnte hier so weit gehen zu vermuten, dass der von uns gezeigte Sprachwandelprozess hier schon viel weiter fortgeschritten ist, da das Suffix -ling als „Patienssuffix” sich nicht durchsetzen konnte und dafür die substantivischen Partizipien II in dieser Funktion generalisiert wurden.

Für -er kommt aus morphologietheoretischer Perspektive hinzu, dass es von der Form her das undeutlichste Derivationssuffix ist; -er hat in der Form unterschiedliche Funktionen, so kann es auch Flexionssuffix (bei Adjektiven oder Plural bei Substantiven) oder Komparationssuffix sein. Für ein Prinzip wie „Wortarterkennung durch Suffixe” ist es also u. U. nicht geeignet, was eine mögliche Motivation für Sprachwandel darstellen könnte.

-chen/-lein ${ }^{4}$ nominalisiert nicht, sein Hauptbereich ist, aus Substantiven neutrale Substantive zu diminuieren - das Blümchen, das Bäumchen, das Stühlchen, das Becherchen. Damit steht es in der Nähe der Flexionssuffixe (Hierarchie nach Eisenberg/Sayatz 2005), dies gilt auch aus typologischer Sicht (cf. Bakema/Geeraerts 2004). Ansonsten folgt ihm kein Pluralsuffix, cf. die Stühlchen, die Blümchen, syntaktisch wohl aber ein Genitivsuffix des Blümchens. Unter Ausschluss von Scherzbildungen (cf. Bauer 2004) ist die Produktivität zwar nach wie vor gegeben, cf. niedrigfrequente Bildungen wie ?Surferchen, ?Chat(ter)chen, ?Blogchen, ?Songchen, ?Internetchen, ?Keyboardchen, so auch historisch, cf. Bildungen wie Ballönchen oder Bonbonchen. Hier scheint es wortspezifische Unterschiede, insbesondere bei Entlehnungen zu geben (cf. Nübling 2013: 46 mit dem Beispiel *Bruttosozialprodüktchen). Hierzu lohnt es, sich den Bezugsrahmen für das Deutsche zu vergegenwärtigen: Im Vergleich zu früheren Sprachstufen und anderen Sprachen (z. B. Italienisch, Russisch) sowie auch den Dialekten (cf. z. B. Schwäbisch, Bairisch, Ostmitteldeutsch) ist die Verwendung der Diminution in der Standardsprache u. E. deutlich seltener geworden, auch in der gesprochenen Sprache. Gegenwärtig lässt sich zwar schwer beurteilen, ob daraus eine zunehmende Unproduktivität der Diminution abzuleiten ist, völlig unwahrscheinlich ist dieses Szenario vor dem Hintergrund der Vergleichsdimensionen „Diachronie”, „Dialekte” und „Sprachvergleich” jedoch nicht. Damit würde sich das Deutsche in diesem Fall ähnlich entwickeln wie einst das Englische, das nur noch Relikte dieser einstigen Produktivität hat (wie bei starlet zu star, booklet zu book oder streamlet zu stream). Zu einer weiterführenden Entwicklung müsste zu der u. E. zu beobachtenden, zunehmend niedrigeren Gebrauchsfrequenz des Deutschen auch die semantische Spezifizierung der Diminutiva hinzukommen (wie bei Liebchen vs. Liebe oder Stövchen). Diese ist jedoch im Deutschen (ggf. bislang) auf wenige Beispiele beschränkt.

-in hat als Movierungssuffix selber nicht die Fähigkeit zur Nominalisierung, sondern es kodiert die Bedeutung des femininen Sexus über die Ableitung von Personenbezeichnungen, insbesondere von genus-maskulinen Individuativa wie Lehrer - Lehrerin, Student - Studentin, Doktorand - Doktorandin sowie von Epicoena wie Hund - Hündin, Ratte - Rättin. Die einzige, formale „Ausnahme” besteht, wenn Schwa das „Suffix” ist wie bei Pole-Polin, Franzose - Französin, Bote - Botin. Letztere Form zur Kodierung von maskulinen Personenbezeichnungen ist aber nicht produktiv, produktiv sind die beiden Nominalisierungsformen der -er-Derivation. Dort affigiert die Motion produktiv sowie der substantivierten Part. I (wie in Studierender) und hier im Speziellen kommt -in gar nicht zum Zuge. Generell ist das Suffix -in ist also kein

\footnotetext{
${ }^{4}$ Im vorliegenden Beitrag wird nur von -chen die Rede sein. Da beide Diminutionsmorpheme einzig über Sprachvariation motiviert sind, treffen die hier getroffenen Aussagen genauso für das Suffix -lein zu.
} 
nominalisierendes Wortbildungssuffix, es modifiziert einzig im Sinne der Sexus-Kodierung. Die Kategorie Sexus ist jedoch derivationsmorphologisch nicht weiter anschlussfähig, was u. a. daran zu erkennen ist, dass movierte Nomina nicht weiter diminuiert werden können (cf. * Lehrerinchen, *Rättinchen). Analog zu -chen steht es damit in einer Suffixhierarchie nah zu den Flexionssuffixen (cf. hierzu Bakema/Geeraerts 2004 aus sprachtypologischer Perspektive). Auch die enge Beschränkung der Basen auf Personenbezeichnungen, nicht auf alle Substantive im Sinne einer domain-specific word-formation, widerspricht auf formaler Ebene der Einordnung als produktives Suffix.

Damit lässt sich zwischenzeitlich zusammenfassen: Feminine Nominalisierungssuffixe verlieren ihre Produktivität (insbesondere -ung und -heit/-keit); die anderen haben ihre Produktivität schon verloren wie -schaft, -nis, -sal usw. Als neutrale Affigierungsmittel stehen -chen und Gee zur Disposition. -chen ist jedoch kein Wortbildungssuffix im strengen Sinne, weil es nicht wortartbestimmend ist. Ge-x-e kann keine Präfixverben ableiten und ist zusammen mit -erei heutzutage auf pejorative Kontexte eingeschränkt (das Gescanne, die Scannerei), beide sind also dermaßen stark restringiert, dass von ihnen keine „Rettung“ der Wortbildung ausgeht.

\section{Substantivische Suffixoide}

Bei substantivischen Suffixoiden könnte man annehmen, dass diese die „Nachfolgegeneration“ von Suffixen darstellen und ihre Grammatikalisierung einem generellen Abbau der Suffigierung entgegenwirkt. Dies zieht die Frage der gegenwärtig produktiven Suffixoide nach sich. Fleischer/Barz (2012: 230f.) diskutieren -wesen, -werk, -zeug. Da sie -zeug als Kompositum interpretieren, kann es nur noch um -werk und -wesen als Suffixe gehen, auch hier zweifeln sie an der Adäquatheit einer solchen Analyse. Altmann/Kemmerling lehnen die konkreten Affixoide als solche ohnehin ab (2000: 54; 102).

Im Einzelnen: -werk nimmt substantivische Basen und bildet Kollektiva wie Schuhwerk. Sind hier auch *Computerwerk, *Technikwerk möglich? Mit verbalen Basen nennen Fleischer/Barz Backwerk. Wie sieht es mit ?Scanwerk, ?Surfwerk, ? Twitterwerk aus? Hier liegen u. E. aufgrund der formalen Beschränkungen Komposita vor (Werk im Sinne von „das Erschaffene”), zum Teil werden auch über den Prozess der Reduktion Analogiebildungen zu bereits existierenden Komposita gebildet (wie bspw. Mailwerk i. S. v. ,Mail-Netzwerk'). Dann wäre -werk eben kein Suffix, sondern Kompositumszweitglied. Analog gilt dies für -wesen: als Kollektivsuffix (Fernmeldewesen, Hochschulwesen) finden sich Wörter, zu denen analog Computerwesen gebildet wird. Computerwesen kann aber auch im Sinne von ,Lebewesen' benutzt werden (wie in hochintelligentes Computerwesen), es meint dann ,eigentliche Natur'; insgesamt also viel zu selbständig, um ein Suffix zu sein. Neue Bildungen finden sich mit -zeug wie Surfzeug, aber es kann paraphrasiert werden als Zeug zum Surfen und ist damit deutlich ein Kompositum. Die Suffixoide für Substantive zeigen nicht wirklich die Tendenz, produktive Suffixe zu werden; einzelne Analogiebildungen sind möglich, aber bei vielen ist das Wort noch zu deutlich und sie sind zu wenig reihenbildend. Die Bildungen sind - neben ihrer niedrigen Frequenz - eher als sog. Scherzbildungen einzuordnen und genügen damit nicht dem hier zugrundegelegten Kriterium der Produktivität (cf. hierzu auch Bauer 2004). Demnach kommen keine neuen Substantivsuffixe durch Grammatikalisierung hinzu. Das heutige Deutsch unterscheidet sich damit grundlegend von früheren Sprachstufen. 
Auch durch Reanalyse (ausführlich cf. Fuhrhop 1998a) sind keine „,neuen” Suffixe als solche zu erwarten - dort werden -ler, -ner,- igkeit, -erei, -lein, -nis diskutiert - lediglich -erei könnte produktiv sein (s. oben).

\subsection{Substantivische Konversion}

Implizite Ableitung/Nullderivation: Im heutigen Deutsch kann quasi alles substantivisch verwendet werden in dem Sinn, dass Kerne der Nominalgruppen gebildet werden. Sinnvoll ist dabei, zwischen morphologischer und syntaktischer Konversion zu unterscheiden. Die morphologische Konversion vom Typ (der) Lauf, zu denen die impliziten Ableitungen wie Wurf assoziiert sind, ist gemäß Distributionsproben mit entlehnten und nativen Verben (wie surfen bzw. bohren) weitgehend unproduktiv, cf. (der) *Surf (zu surfen), (der) *Chill (zu chillen), (der) *Bohr (zu bohren).

Der substantivische Infinitiv: das Laufen, das Werfen, das Surfen, das Chillen ist als eine syntaktische Umkategorisierung hochproduktiv. Auch formal ist er restriktionsfrei - sowohl Präfix- als auch Partikelverben sind hier gleichermaßen möglich: cf.: das Laden, Beladen/Ausladen. Auch (Rektions-)Komposita sind möglich wie Möbelausladen. Und er funktioniert bei fremden Basen ebenso: das Squeezen das Zersqueezen/das Wegsqueezen/das ZahnpastatubenAussqueezen.

Ein deutlicher formaler Unterschied zwischen der oben erwähnten morphologischen Konversion und der syntaktischen Konversion kann an der Form der „Attribute” gesehen werden: Im Gegensatz zu suffixalen Nominalisierungen können Verbalphrasen sogar Adverbiale mitnominalisieren, cf. auch Abschnitt 3.3: die gründliche/*gründlich Beobachtung vs. das gründliche Beobachten/das Gründlich-Beobachten. Dass das Adverbial (hier gründlich) bei der Transposition erhalten bleibt, zeigt, dass der verbale Charakter des Infinitivs zum Teil erhalten bleibt, da in herkömmlichen Nominalphrasen Adjektive nicht unflektiert bleiben: *die ständig Pause/*Ständigpause, aber die ständige Pause (für weitere Details der syntaktischen Herleitung cf. Alexiadou et al. 2011).

Dazu passt auch, dass unflektierte Adjektive (wie hammer, klasse) ausschließlich mit dem substantivischen Infinitiv nominalisiert werden können und daher deadverbiale Bildungen wie Hammersein (cf. bspw.: $x$ tanzt hammer/klasse/...) möglich sind im Vergleich zu formal unmöglichem *Hammerheit. Demzufolge ist die Restriktionslosigkeit der Konversion im Vergleich zur Derivation ein wichtiges, wenn nicht sogar das wichtigste Argument in Bezug auf die zunehmende, synchrone Produktivwerdung des Bildungsmusters.

Wesentliche Unterschiede zwischen beiden Nominalisierungsmustern sind, dass die syntaktische Konversion keineswegs vollständig passieren muss. So ist bekannt, dass produktiv gebildete substantivische Infinitive keine Pluralform bilden, sehr wohl aber nach Kasus flektieren (des Laufens, *die Laufen). Die typischen, substantivischen Adjektive wie der Angestellte flektieren weiterhin wie Adjektive (der Angestellte - ein Angestellter) und bilden entsprechend den Plural (die Angestellten). Ebenso verhalten sich die substantivischen Partizipien I: der Reisende - ein Reisender, der Studierende - ein Studierender. Als Kerne der Nominalgruppen sind Pluralformen häufig möglich wie die Ichs, die Grüns, die Blaus, die As - auffällig ist aber die 
eindeutige Bevorzugung des s-Plurals in diesem Bereich, nicht *die Iche, *die Grüne usw. Wie beim substantivischen Infinitiv ist auch hier das Neutrum das Genus der Wahl.

\subsection{Zwischenfazit: Substantivische Ableitung}

In den Wortbildungen werden -heit/-keit, -er, -ung, -erei/Ge-...-e, -chen und -in als produktive Suffixe (bzw. im Falle von Ge-...-e als Zirkumfix) genannt. Die Bildungsmuster mit -erei bzw. $G e-\ldots-e$ sind von vorneherein in ihrem nominalisierenden Wirkungsbereich stark eingeschränkt, da sie im heutigen Deutsch produktiv nur noch pejorativ verwendet werden. -chen und -in sind vor dem Hintergrund der Kriterien von Transposition und Modifikation an sich keine Wortbildungssuffixe, da mit ihnen keine neuen Wörter im strengen Sinne gebildet, sondern lediglich modifiziert werden.

Es bleiben -heit/-keit, -ung, -er: Für -ung hat bereits Demske (2000) gezeigt, dass es in seiner Produktivität abnimmt und durch den substantivischen Infinitiv verdrängt wird. Der Infinitiv wird über syntaktische Konversion zu einem Substantiv. Für -heit/-keit und -er scheinen sich Alternativkonstruktionen ins System zu drängen, die beide aufgrund des Status als syntaktische/grammatische Transposition (unmarkierter Wortartwechsel) keine Wortbildungen im strengen Sinn sind: So wird -heit/-keit durch ein sein-Kompositum „,bedroht”-Famesein, Highsein, Coolsein, aber auch Verliebtsein, Betrunkensein. Auch dieses ist eine syntaktische Konversion.

Möglicherweise ist das Suffix -er durch substantivische Partizipien bedroht wie der Lehrende, der Reisende, der Vorsitzende. In einem Agens-Patiens-Verhältnis ist das ganz interessant, denn -ling ist für das Patiens (Prüfling) schon lange nicht mehr produktiv, sondern der Geprüfte, auch dies eine syntaktische Konstruktion. Musterbildende Neuzugänge durch Suffixoide konnten wir nicht finden.

\section{Das Adjektiv}

\subsection{Adjektivische Suffigierung}

Zahlreiche adjektivische Suffixe sind im Gegenwartsdeutschen nicht mehr produktiv (wie bspw. -sam und -lich). Im Folgenden sollen die für das Gegenwartsdeutsche mutmaßlich als produktiv geltenden Suffixe diskutiert und in Bezug auf eine mögliche Unproduktivität vor dem Hintergrund der Syntaktisierung geprüft werden.

Das Suffix -ig ist nur noch bei Zusammenbildungen produktiv wie siebenstöckig, zweibildschirmig, kleinwagig (cf. Fleischer/Barz 2012: 337), hartkernig (ebd.). Außerdem gilt -ig als produktiv für die Umkategorisierung von Adverbien wie heutig, jetzig, hiesig, dortig; in diesem Sinn auch Fleischer/Barz (2012: 339) rückwärtig, nachherig, sofortig, damalig. Ob das Suffix für Adverbien jedoch wirklich produktiv ist, ist anhand von Distributionsproben mit nicht-nativen Stämmen (s. Abschnitt 1) zu ermitteln, wie an Wörtern wie super oder top zu erkennen ist: Die prädikative Verwendung (Rom war super/top) und adverbiale Verwendung (Hans malt super/top) war im Deutschen die primäre; die spätere, attributive Verwendung erfolgt unmar- 
kiert: ein super/*superig Spiel bzw. ein top/*top(p)ig Spiel. Eine Produktivität ist für das Suffix -ig zumindest für diesen Bereich nicht nachzuweisen. Stattdessen finden sich in adverbieller Funktion hier Argumente für eine syntaktische Umkategorisierung: Auch andere lexikalische Elemente wie Nomina (cf. donnerstag) können ohne formales Hilfsmittel verwendet werden (er kommt donnerstag), in einer ggf. ehemaligen Konkurrenz zu donnerstagig, donnerstäglich (wie sonntäglich), s. Abschnitt 3.3. Auch wenn u. U. das Suffix noch in einigen Fällen, v. a. im mündlichen Sprachgebrauch, anzutreffen ist (wie ein sackiger Mantel, ein Mantel wie ein Sack', ein buchiges Heft , ein Heft wie ein Buch'), so scheint hier die adjektivische Affixoidbildung in attributiver und prädikativer Verwendung kontinuierlich zuzunehmen, cf. ein sack-mäBiger Mantel, ein buch-artiges Heft bzw. $x$ ist sackmäßig/buchartig.

-bar ist damit das einzige deverbale Adjektivsuffix, was als solches noch produktiv ist. -bar ist aber insofern in der Wortbildung markiert, als es auf eine klare, $d$. h. syntaktische beschreibbare Basis anwendbar ist, nämlich auf transitive Verben. In den 80er Jahren, als viele versucht haben, die Wortbildung mit syntaktischen Mitteln zu beschreiben, wurde dies häufig an dem Suffix -bar gezeigt (cf. Höhle 1982; Reis 1983; Lieb 1983) - es kann als das „syntaktischste” unter den bekannten Wortbildungssuffixen gelten. Es ist auch heute noch produktiv: scannbar, screenbar, laserbar, downloadbares, mailbar, möglicherweise wird es in einigen Fällen durch -fähig ,, ersetzt” wie in biegefähig, zitierfähig (cf. Fandrych 2011: 145). Das Suffix ist in prädikativer Funktion u. E. aber auch durch $z u+$ Infinitiv-Konstruktionen ersetzbar (die Schrift ist lesbar $\rightarrow$ die Schrift ist zu lesen, die Schrift kann man lesen'), was aufgrund der synchronen Syntaktifizierbarkeit zeigt, dass auch dem Suffix eine (morpho-)syntaktische Grundfunktion zukommt. Eine langfristige Ersetzbarkeit durch analytische Konstruktionen wäre also formal möglich. Die Annahme einer möglichen Syntaktifizierbarkeit ist, neben der bereits bestehenden syntaktischen Funktion der Derivation transitiver Verben, auch deswegen plausibel, da das zu -bar historisch in sog. ,passivischer Funktion“ in Konkurrenz stehende und einst produktive Adjektivsuffix -lich (wie z. B. unvergleichlich/unvergleichbar, cf. Wilmanns 1896: 495) in attributiver Verwendung durch $z u$ + Präsenspartizipien (Typ: ein zu lesendes Buch) ersetzt wurde (cf. Wilmanns 1869: 410; 509). Vor dem Hintergrund der Diachronie wird ersichtlich, dass Syntaktisierungs- und Syntaktifizierungstendenzen nicht erst im Gegenwartsdeutschen begonnen haben, sondern deutlich älteren Datums sind.

-haft gilt (neben den Suffixoiden -mäßig und -technisch) als das einzige desubstantivische Adjektivierungssuffix des Deutschen, das im desubstantivischen Bereich gegenwärtig noch produktiv ist. Allerdings deriviert es, im Gegensatz zu früheren Sprachstufen (cf. lebhaft, lachhaft, aber *chatthaft, *surfhaft), ausschließlich Substantive (wie sonnenhaft, computerhaft, regalhaft), insbesondere pluralisierte Personenbezeichnungen (cf. studenten-haft, professoren-haft), sogar movierte (wie in chefinnen-haftes Benehmen). Allerdings können -haft-Bildungen stets durch das Suffixoid -mäßig ersetzt werden: standardhafter Einbau $\rightarrow$ standardmäßiger Einbau, ?lampenhafte Ausstattung $\rightarrow$ lampenmäßige Ausstattung, usw.

Die Affixoide bei den Substantiven haben wir als für unsere These nicht relevant beschrieben (s. oben). Bei den Adjektivsuffixoiden mag das anders sein. In dem Aufsatz „Wie geht es eigentlich den „Halbsuffixen”?“ (cf. Fandrych 2011) geht es interessanterweise ausschließlich um adjektivische Halbsuffixe. Insgesamt sieht Fandrych hier mit Eichinger (2000) das Füllen verschiedener Lücken; für die vorliegende Argumentation kann man grob zusammenfassen, dass sie das weitgehende Fehlen von (produktiven) Adjektivsuffixen aufheben.

ISSN 1615-3014 
Fandrych prüft dies für die verschiedenen semantischen Muster (nach Eichinger 2000); hier kann dann zum Beispiel für ein Muster ,Y kann ge-X-t werden' neben -bar und vereinzelten anderen Bildungen wie begreiflich, biegsam, unglaublich, unaufhaltsam, unverkennbar auch fähig genutzt werden (biegefähig, zitierfähig). Insgesamt sieht Fandrych also die adjektivische Wortbildung in ,gebundenen” Zweitgliedern und freien Zweitgliedern (also letztlich Komposition) gut aufgehoben; Fandrych erkennt hier ein produktives Wortbildungsmuster, das am ehesten als „Rektionskomposition” zu bezeichnen ist. Dieses Muster überträgt sich auch auf solche Zweitglieder, die nicht von sich aus Rektion haben. Die diskutierten gebundenen Zweitglieder sind -freudig, -pflichtig, -haltig, -artig, -förmig, -widrig. Daneben diskutiert er zahlreiche Zweitglieder wie gleich, gemäß, getreu, intern, nah, fähig, lustig, aktiv, froh, willig, süchtig, bereit, verrückt, faul, müde usw. Auf dem Weg, in einem bestimmten Sinne prototypische Wortbildungssuffixe zu werden, sind sie nicht. Letztendlich kann man das folgendermaßen im Sinne der hier verfolgten Fragestellungen interpretieren: Hier entsteht ein Konstruktionstyp der adjektivischen Rektionskomposita, der produktiv ist. Interessant für unsere These ist, dass ein Typ entsteht, der eben gerade nicht an eine bestimmte formale Einheit gebunden ist, sondern mit unterschiedlichen Einheiten funktioniert.

\subsection{Konversion bei Adjektiven}

Unverkennbar werden die Partizipien in attributiver Funktion genutzt und hier flektiert wie Adjektive. Adjektive können daneben prädikativ und adverbial benutzt werden. Das Partizip II kann prädikativ genutzt werden - es ist die Form, die als Zustandspassiv diskutiert wird (cf. Maienborn 2007, Rapp 1996). Unabhängig von der grammatischen Interpretation sind Formen wie der Kuchen ist gebacken, das Buch ist gelesen und das Problem ist gelöst grammatische Formen des Deutschen. Das Partizip I tritt hingegen nicht prädikativ auf *die Kinder sind singend (cf. Fuhrhop/Teuber 2000; s. aber Lübbe 2013). Adverbial sind beide möglich, und zwar auch mit entsprechenden Ergänzungen wie (altmodische Schlager) singend fuhr sie zur Uni, (von den Kollegen) genervt schlug sie die Tür zu.

Beide sind von jedem Verb bildbar; das Partizip II ist nicht durchweg als Adjektiv gebräuchlich wie * die gefahrenen Studenten, *der umgezogene Professor (cf. Eisenberg 2013c: 101), kann aber durch einen telischen Kontext dazu gemacht werden die nach Bonn gefahrenen Studenten, der nach Berlin umgezogene Professor oder die gefahrene Strecke. Dies spricht aufgrund der notwendigen Miteinbeziehung analytischer, d. h. syntaktischer Realisationen für eine morphosyntaktische Analyse und ist somit im Sinne der Syntaktisierung zu interpretieren. Es gibt diverse Unterschiede zwischen den Partizipien: Das Partizip II kann Bestandteil von Verbformen sein (Perfekt, Passiv), das Partizip I kann das im heutigen Deutsch nicht. Andererseits kann das Partizip II eher mit un- (ein typisches Adjektivmerkmal) präfigiert werden (ungelesen, ungelöst, unausgeschlafen), das Partizip I kann das nicht ( *unsingend, *ungewinnend, *unspielend). Das Partizip I verhält sich insgesamt einheitlicher, das Partizip II vielfältiger. Nichtsdestotrotz handelt es sich weder um Wortbildung (Partizip I, wo ja immerhin das -(en)d so interpretiert werden könnte) noch um morphologische Konversion, sondern in beiden Fällen um syntaktische Konversionen. 
Dass das -(en) $d$ kein Wortbildungssuffix ist, steht schon (indirekt) bei Bech (1955) und ist besonders auch an Formen des modalen Passivs zu sehen: das zu lösende Problem (das Problem ist zu lösen).

\subsection{Klasse, hammer, donnerstag}

Bisher haben wir unter den Affixoiden die Suffixoide betrachtet. Präfixoide sind solche wie Höllen-, Affen-, Bomben-, Klasse-, Spitze- in Höllenangst, Bombenerfolg, Klasseweib, Affenangst, Spitzensound (cf. Gaeta 2010: 115). Ein Argument ist ja, dass neben Höllenangst auch Höllenfreude möglich ist, die Ursprungsbedeutung ist deutlich abstrahiert.

Eine mögliche Entwicklung ist die folgende, wie bei dem Satz Die Jacke ist aus strick zum primären Substantiv Strickjacke erkennbar: Die Bedeutung von Erstgliedern in Komposita wird allgemeiner und abstrakter, so wie das (mit dem gleichzeitigen Eintreten von Reihenbildung) bei der Entstehung von Präfixoiden der Normalfall ist. Ohne Reihenbildung und lexikalische Desemantisierung entwickeln sie sich aber nicht zu Präfixen, sondern werden - mit ihrer verallgemeinerten lexikalisch-abstrakten Bedeutung - selbstständig, zumindest gilt dies für einige: das war spitze, hölle, klasse $e^{5}$ und entsprechend wieder das klasse Auto. Diese Bestandteile werden also über die Zwischenstufe „Präfixoid” nicht zum Präfix, sondern sie werden wiederum zu selbstständigen Wörtern. ${ }^{6}$

In diesen Bereich gehören wohl auch Konstruktionen wie das ist ja hammer und auch bei Entlehnungen wie die ist voll fame. Solche Konstruktionen sind sicherlich eher typisch für die gesprochene Sprache (und hier auch für die Jugendsprache). ${ }^{7}$ Da die gesprochene Sprache, insbesondere die Jugendsprache, sich stärker als die geschriebene existierenden Normierungen bzw. Normierungsversuchen widersetzt, lassen sich hier besonders deutlich sprachliche Innovationen für die spätere Standardsprache beobachten, welche u. E. kompatibel mit dem Konzept der Syntaktisierung sind.

\footnotetext{
${ }^{5}$ In der prädikativen Funktion ist die Wortart strukturell ambig - er ist grün, er ist Lehrer; eher geht es in Richtung Adjektiv; Artikel sind nicht (ohne Bedeutungsänderung) möglich die Sendung war die Spitze $\neq$ die Sendung war spitze. Hier wäre also ein Umweg beschrieben über Präfixoide. Ohne diesen Umweg funktionieren er ist pleite, er ist schuld $\neq$ er ist die/eine Pleite, er ist die/eine Schuld.

${ }^{6}$ Ein vergleichbarer Prozess findet sich auch bei Konfixen - so finden sich durchaus Konstruktionen wie Salat esse ich nur bio; er denkt öko, er ist ein Öko, s. auch Buchmann (2015).

${ }^{7}$ Wenn hammer ein Adjektiv wäre, wäre *Hammerheit denkbar, das geht wohl nicht, Hammersein wird als „,besser" bewertet. Denn durch die gesteigerte Produktivität des nominalisierten Infinitivs ist auch die Möglichkeit zur Propositionalisierung von Adverbien gegeben, was sonst so vorher nicht ging, cf.: *Heute-heit, aber Heut-ig-keit (mit Tendenz zur Lexikalisierung), dafür (das) Heute-Sein, *Hier-heit, aber Hies-ig-keit, aber auch das Hier-Sein. Der einstige „Umweg“ über das Adjektiv fiele bzw. fällt also weg. Das würde passen zu der These, dass -heit bereits an Produktivität einbüßt - zumindest widerspricht die Nichtbildbarkeit von *Hammerheit keinem der hier genannten Argumente. Parallel dazu würden auch die desubstantivischen -haft-Nominalisierungen (wie Professorenhaft-igkeit) in derselben Bedeutung durch Infinitivnominalisierungen ersetzt werden können: das ProfessorSein. Auch hier fiele der „Umweg“ über die Adjektivderivation weg.
}

ISSN 1615-3014 


\section{Die Adverbkennzeichnung im heutigen Deutsch}

Im Englischen und Französischen (und vielen anderen Sprachen) werden Adverbien extra gekennzeichnet, durch -ly bzw. -ment usw. Im Deutschen gibt es einige wenige Adverb"suffixe", und zwar $-s$ und -erweise (z. B. Fuhrhop 1998a). Diese sollen im Folgenden Gegenstand der Diskussion sein.

Das Suffix -erweise ist an sich schon hochmarkiert, man erkennt die Syntax sehr deutlich wie in verständlicherweise - in verständlicher Weise. Zudem fungiert es ausschließlich als Satzadverbial, nicht als Modifikator zu Nominalgruppen, Adjektivgruppen oder Verbalphrasen, wie der „es ist $x$, dass“-Test beweist: Verständlicherweise geht er ins Kino $=$ Es ist verständlich, dass er ins Kino geht.

Das Adverbsuffix -s wie in nachts, sonntags, flugs, mittags und auch in eilends, durchgehends, zusehends, besonders (cf. Fleischer/Barz 2012: 369) ist durchsichtig, Produktivität ist aufgrund der Distributionsbeschränkungen nicht mehr zu erkennen, cf.: (Er telefoniert) *chatrooms/*herummailends oder in (Er tanzt) *ferientags/*holidays/*saturdaynights. Daraus ergibt sich eine diachrone Abnahme der adverbialen Suffigierung und eine Zunahme der Konversion im Sinne einer Syntaktisierung.

In diesem Sinne kann man auch Eisenberg (2002) lesen: Wenn man von den drei typischen syntaktischen Funktionen des Adjektivs ausgeht, also attributiv (zum Substantiv), adverbial (zum Satz oder Verb) und prädikativ, kann man feststellen, dass nicht alle Adjektive gleichermaßen für alle Funktionen taugen. So trägt grün in er denkt grün eine übertragene Bedeutung, schnell ist in er läuft schnell völlig unmarkiert und in das schnelle Auto eher sekundär. Viele bar-Adjektive sind adverbial eher markiert? er kocht essbar; prädikativ und attributiv hingegen unmarkiert (das Wasser ist trinkbar, das trinkbare Wasser). Adjektive beziehen sich im adverbialen Gebrauch eher auf Verben, im attributiven oder prädikativen auf Substantive: Aus diesem Grund ist die Aufteilung nicht so überraschend. Allerdings kann das Partizip I zwar attributiv und adverbial, nicht aber prädikativ verwendet werden (singend kam er um die Ecke, die singenden Kinder - *er ist singend). Die These ist die folgende: Adjektive kommen primär nicht in allen drei Funktionen vor; so kommen manche attributiv und prädikativ vor (grün), andere attributiv und adverbial (Partizip I); Adverbien sind dann die Adjektive, die prädikativ und adverbial vorkommen; wenn Adjektive aber nicht attributiv vorkommen, brauchen sie nicht flektierbar zu sein. Adverbien sind unflektierbar, weil sie in der einzigen Funktion, in der flektiert werden müsste, nicht vorkommen. Historisch musste zur Adjektivierung, d. h. um Flektierbarkeit herzustellen - wie oben in Abschnitt 3.1 angedeutet - ein -ig (dortig, heutig, hiesig, cf. jedoch *coolig, *surfig) eingefügt werden.

Daraus kann man Folgendes schließen: Man unterscheidet vermeintlich Wortarten auf lexikalischer Ebene, obwohl man etwas anderes, nämlich Syntax bzw. Morphosyntax, meint. Im Vergleich zum Englischen ist hier dann ein wesentlicher Unterschied zu erkennen: Wo das Deutsche syntaktisch die (Nicht-)Flexion zur Unterscheidung hat, wählt das Englische morphologisch ein Suffix (zur Diachronie cf. Pounder 2001). Der einzige Unterschied ist dann der prädikative Gebrauch: Hier wählt das Deutsche die unflektierte Form - eine Formgleichheit zum adverbialen Gebrauch. Das Englische wählt die Form ohne -ly und rückt es damit in Vergleichbarkeit zum attributiven Gebrauch. 


\section{$5 \quad$ Das Verb}

Im Sinne einer möglichen Syntaktisierung ist auch die verbale Wortbildung auf eine mögliche Produktivität hin zu diskutieren. Als verbale Suffixe sind -eln, -ern und -ieren ererbt, jedoch nicht (mehr) in vollem Umfang produktiv (cf. *maileln/*mailern/*mailieren). Stattdessen ist die verbale Konversion für die Verbbildung das Mittel der Wahl. Das Suffix -eln ist inzwischen pejorativ (laufeln, schreibeln), -ern ist in keinem Fall mehr produktiv und -ieren als Integrationssuffix ist auch nicht mehr produktiv. Die Endung selbst ist nicht unmittelbar aus einer französischen Verbendung entstanden, denn die Verben wie frisieren oder frittieren sind auf Französisch friser bzw. frit > frire, frittieren'.

Verbale Präfixe sind als nicht-wortartverändernde Präfixe noch zum Teil produktiv wie (vermailen - ,falsch mailen', Zeit vermailen, ,verschicken'), vertwittern, verfacebooken. An dieser Stelle wäre auch vor dem Hintergrund der Diachronie zu fragen, ob und inwieweit man Präfixen einen „echten” Wortartänderungsstatuts zubilligen kann, da zumindest bei den von uns betrachteten Entlehnungen kein solcher Effekt erzielt wird (cf. die Simplex-Korrelate mailen, twittern, facebooken). Da Präfixe nicht wortartverändernd sind, ist aus ihnen auch keine grammatische Relevanz in Bezug auf die Fähigkeit zur Transposition, einer der Grundfunktionen der derivativen Wortbildung, abzuleiten. Darüber hinaus ist die Reihenbildung von Präfixen zu großen Teilen diskutabel: So ist bspw. das Präfix ent- oder ver- zwar noch mit Entlehnungen kombinierbar (er entsurft sich/es entsurft ihm, er entsurft ihr... er entsurft der Realität??), allerdings sind Bildungen dieser Art außerordentlich niedrigfrequent (?entmailen, ?entsurfen, auf Google jeweils unter 20 Treffer, Stand 16.11.2015) und wirken stilistisch markiert, d. h. so, als wäre das jeweilige Verb metaphorisch in Analogie zu einem bereits präfigierten Verb verwendet (entmailen statt entsenden, entsurfen statt entschwinden). Zudem sollten Affixe im Sinne der Wortartenspezifizität (domain-specific word formation) mit allen verbalen Entlehnungen kombinierbar sein (Kriterium der Restriktionslosigkeit). Bei einigen Entlehnungen führt dies jedoch teils zu fragwürdigen Ergebnissen (wie sie ?ver-/er-chillt), so dass man abermals keine unmarkierte, reihenbildende Produktivität, sondern eher wieder Scherzbildungen annehmen kann, die im Sinne von Bauer (2004) zur Ermittlung von Produktivität in Analogie zu bereits existierenden Bildungen (im nominalen Bereich wäre dies bspw. ?Schönsal wie Scheusal; ?Twitterant wie Informant) auszuschließen sind.

Partikelverben wie anmailen, ansurfen (Internetbelege) sind dafür hochproduktiv; ein deutliches Beispiel ist die Reihenbildung mit mit-: mitarbeiten, mitputzen, mitsingen, mitschreien, mitscannen, mitcoachen. Diese Partikelverben sind aber aufgrund ihrer syntaktischen Distribution und ihrer Semantik nicht mit unselbstständigen Affixen gleichzusetzen, cf. auch Lüdeling (2001). Auch Olsen (z. B. 1996: 274) zeigt, wie sie sich aus der Syntax herausbilden (Sie trug Feuchtigkeitscreme (auf ihre Haut/ihrer Haut) auf). Sie werden damit zwar relativ morphologischer, es sind aber keine genuin morphologischen Strukturen. So bleiben sie auch trennbar (er putzt mit - *er mitputzt).

Zum anderen verbinden sich echte Verbpartikeln ohne weiteres mit fremden Verbstämmen jeder Art, z. B. andiskutieren, abisolieren, durchpowern, wegmanagen, umbandagieren, übercollagieren. In der Unbegrenztheit von Hybridbildungen dieser Art spiegelt sich die relative Unabhängigkeit der Verbpartikel vom Stamm, die ja in der Trennbarkeit ihren stärksten Ausdruck findet.

(Eisenberg 2012: 296) 
Im verbalen Bereich gibt es statt Derivationen besonders die Konversionen, in denen Wörter als Verben benutzt werden - insbesondere anhand der Flexion wird dann die Verbalität sicht- und hörbar: er twittert, er mailt/emailt, wir facebooken den Termin, doodeln, googeln, skypen usw. Auch Rückbildungen gehören in diesen Bereich. Rückbildungen sind möglich von Bildungen wie eislaufen, brustschwimmen, aber auch downloaden usw. Mitunter entstehen sie mit unvollständigen Paradigmen wie bspw. bauchtanzen - ?bauchgetanzt/?gebauchtanzt. Man kann letztere sog. Rückbildungen folgendermaßen interpretieren: Sie sind stets als Verben gekennzeichnet - durch eine Infinitivendung (bauchtanzen, kunstturnen, bausparen), durch eine Partizipkennzeichnung, wenn sie denn möglich ist (bauchgetanzt, gebauchttanzt, kunstgeturnt, gekunstturnt, baugespart, gebauspart) oder durch eine finite Endung (weil sie bauchtanzt/kunstturnt/bauspart). Problematisch ist die syntaktische Trennbarkeit - *sie tanzt bauch; untrennbar sind sie aber auch nicht *sie bauchtanzt. Im Sinne der vorliegenden These zeigt sich auch hier, dass die Flexion (Morphosyntax) eine funktionale Ausweitung zulässt; sie hat ihre Grenzen (noch) an den ,undeutlichen” Positionen. Im Prinzip sind diese Formen also auch ein Typ der ,syntaktischen” Konversion, jedoch aufgrund der unvollständigen Paradigmen eher als eine sekundäre Syntaktisierung zu fassen, da ihnen nominale Komposita zugrunde liegen (wie Bauchtanz, Kunstturner, Bauspar-er/-vertrag). Ihre morphosyntaktischen Beschränkungen zeigen deutliche Analogien zu primären Formen der Syntaktisierung, wie bspw. die Nicht-Pluralfähigkeit des substantivischen Infinitivs, s. Abschnitt 2.2.

\section{$6 \quad$ Wandel in der Fremdwortintegration}

Bis ins 19. Jahrhundert ist eine Tendenz zur Integration mit (zusätzlichen) Suffixen festzustellen, die eine Zuordnung eindeutiger machen. Am deutlichsten ist das bei den Verben mit dem Suffix -ier(en); auch mit dem Adjektivsuffix -isch ist die Tendenz zu erkennen:

\begin{tabular}{|l|l|l|}
\hline & Bis ins 19. Jahrhundert & 20./21. Jahrhundert \\
\hline Adjektive & $\begin{array}{l}\text { musikalisch, amerika- } \\
\text { nisch, genialisch }\end{array}$ & cool, fit, hip, pink \\
\hline Verben & $\begin{array}{l}\text { frisieren, frittieren, de- } \\
\text { monstrieren, trainieren }\end{array}$ & $\begin{array}{l}\text { joggen, scannen, screenen, } \\
\text { coachen, kiten }\end{array}$ \\
\hline
\end{tabular}

Tabelle 1: Wandel der Integration von Adjektiven und Verben

Fremdwörter tragen also ein „Integrationssuffix”, das eindeutig die (lexikalische) Wortart zeigt, jenseits der Flexion. Hier könnte man die gleiche Tendenz bei einigen Personenbezeichnungen vermuten: So findet man einige „Doppelmarkierungen” mit -er (wie in -an-er statt*Amerikan, Kassierer statt Kassier; ebenso Rebeller, Barbierer, Rentenierer (cf. Fleischer/Barz 2012: 206). Auch -ist verschwindet bei einigen Substantiven wieder, die durch ihr prosodisches Muster genügend gekennzeichnet sind wie Baritonist, Tenorist, Sopranist(in), Altist(in), Theologist, heute Bariton, Tenor, Theologe (cf. auch Fuhrhop 1998a: 117; Köpcke 1995). Im 20. Jahrhundert scheint die Tendenz zu dieser eindeutigen Markierung sozusagen nicht mehr ,nötig”. Man mag jetzt argumentieren, dass das im Wesentlichen mit der Hauptgebersprache zusammenhängt, aber trainieren wurde im 19. Jahrhundert eben auch mit einem Suffix versehen. Es geht sogar 
so weit, dass derartige Suffixe bei Anglizismen bei der Übernahme ,verschwinden” wie *Scientologist; Scientologe ist die gängige Form.

Noch heute ist an einigen Beispielen eine „Integrationssuffigierung” festzustellen (cf. Fuhrhop 1998b); Fremdwörter tragen ein natives Suffix, das zum Beispiel eindeutig die Wortart festlegt, jenseits der Flexion. So finden sich amerikanisch, Amerikaner (amerikanisieren), während *amerikan kein mögliches Wort des Deutschen ist. Ebenso Wörter wie musikalisch (*musikal), physikalisch. Daneben gibt es aktiv/Aktiv, aber auch Konjunktiv - konjunktivisch. Bei solchen Adjektiv-Substantiv-Paaren gäbe es quasi drei Möglichkeiten: Beide werden eindeutig gekennzeichnet, eines wird gekennzeichnet oder keines. Im heutigen Deutsch wäre entsprechend Fame - fame (oder Fashion - fesch, für Substantiv und Verb Layout-layouten).

Besonders bekannt ist das Verbsuffix -ieren, dessen Hauptfunktion die Integration war mit dem bekannten Nebeneffekt, dass -ieren einen Akzent auf sich zieht.

\section{Parallele zur Substantivgroßschreibung resp. der satzinternen Großschreibung}

Die Graphematik kann als „Spiegel” der Morphologie und der Syntax gefasst werden. Als solche, so wäre zu erwarten, sollten somit morphologische und syntaktische Entwicklungen irgendwann auch in der Graphematik Abbildung finden. Tatsächlich lassen sich historische Entwicklungen feststellen, die mit dem Konzept der Syntaktisierung kompatibel sind. Diese sollen im Folgenden skizziert werden.

Im 20. Jahrhundert hat sich die satzinterne Großschreibung endgültig von der lexikalischen Bestimmung zu einer syntaktischen hin verändert (Substantivgroßschreibung zur satzinternen Großschreibung). Das erkennt man daran, dass vermeintliche Substantive, die in einer bestimmten Umgebung ihre prototypischen Substantiveigenschaften einbüßen, nicht mehr großgeschrieben werden (er ist schuld, pleite), ebenso wie oben erwähntes das ist hammer.

Bredel (2006: 154f.) interpretiert den bekannten Wandel in der Substantivgroßschreibung folgendermaßen: Zunächst gab es die Anredegroßschreibung der Höflichkeit, dies ist die pragmatische Großschreibung, die sich ausweitet auf Nomina sacra. Die Ausweitung auf Eigennamen und schließlich auf Konkreta beschreibt sie als semantisch. Von Konkreta auf Abstrakta entsteht eine lexikalische Ausweitung. Das ist der Stand am Ende des 19. Jahrhunderts. Zu Beginn des 20. Jahrhunderts dann - und das ist der hier interessante Schritt - erfolgt der Wandel von einer lexikalischen Großschreibung hin zu einer syntaktischen, die sich darin zeigt, dass vermeintliche Substantive (Substantive in einem lexikalischen Sinn wie Schuld, Pleite usw.) in bestimmten Kontexten wieder klein geschrieben werden, weil sie hier nicht in Nominalgruppen auftreten (können) wie er ist pleite $\neq$ er ist dieleine Pleite, er ist schuld $\neq$ er ist die Schuld.

In der modernen Didaktik spricht man deswegen auch nicht mehr von Substantivgroßschreibung, sondern von satzinterner Großschreibung - sie ist also syntaktisch geregelt. Gerade verschwinden die letzten Reste von einer eindeutigen Markierung von Substantiven (-ung, -heit), zumindest produktiv - das Deutsche vollzieht dann den Weg, dass „Substantiv” oder die „Großschreibung" nicht lexikalisch zu fassen ist (gar nicht mehr), sondern ausschließlich syntaktisch. 


\section{$8 \quad$ Zusammenfassung und Ausblick}

Wir haben gezeigt, dass sich die Suffigierung vor dem Hintergrund ihrer bisherigen Entwicklung nicht im selben Maße fortsetzt. Der Vergleich der Derivationsmuster nach Wortarten getrennt betrachtet, zeigt Folgendes: Bei den Substantiven ist -er produktiv, d. h. ein Suffix, das formal schwer von der Flexion unterscheidbar ist. Ansonsten ist für die Substantivierung die syntaktische Konversion typisch (das Ich, das Grün, das Singen) und der Singende - der Geliebte. Bei den Abstraktbildungen verliert das Suffix -ung bereits seit frnhd. Zeit an Produktivität (cf. Demske 2000; Werner 2010), stattdessen wird auch hier der Infinitiv gesetzt (?Surfung $\rightarrow$ das Surfen). Wenn diese Entwicklung so weitergeht, würden -heit-Bildungen mit departizipialen und deverbal-adjektivischen Basen zunehmend von Infinitivnominalisierungen beginnen ersetzt zu werden (die Verspanntheit $\rightarrow$ das Verspannt-Sein; die Verfügbarkeit $\rightarrow$ das Verfügbar-Sein). Sollte diese Entwicklung sich fortsetzen, so wären (natürlich ohne dabei teleologische Sprachwandelmodelle bemühen zu wollen) als wahrscheinliche Folge (irgendwann) auch die adjektivischen Basen betroffen. Sie würden dann v. a. in Analogie zu den Partizipien in Form von substantivischen Infinitiven mit Kopulaverben realisiert werden: die Schönheit $\rightarrow$ das Schön-Sein, ebenso wie das Coolsein, das Famesein. Damit wäre im substantivischen Bereich mit Ausnahme des Suffixes -er die Suffigierung vollständig von der Konversion abgelöst. Und auch für -er zeigt sich schon ein analoger Prozess mithilfe der substantivischen Partizipien I - der Reisende, der Lehrende, der Prüfende.

Bei den Adjektiven findet sich unter den Suffixen noch das Suffix -bar, welches aufgrund seiner grammatischen Funktion in der Literatur auch als ein syntaktisches Suffix beschrieben wurde und damit grammatisch bedeutsam ist. Daneben wäre historisch noch das Suffix -ig zu stellen, welches Adverbien zu Adjektiven macht (cf. heute $\rightarrow$ heutig). Das Suffix -ig deriviert aber im heutigen Deutsch produktiv nur noch Nominalphrasen (cf. siebenstöckig, kleinwagig zu den NPs sieben Stöcke, kleiner Wagen), nicht aber mehr nur Substantive (cf. *stöckig, *wagig). Ein weiteres Argument ergibt sich aus der Tatsache, dass diachron alle Suffixe (adjektivisch und substantivisch) im Gegensatz zu früheren Sprachstufen eine Wortartenspezifik entwickelt haben (zum Substantiv cf. Werner 2012; zur synchronen Systematik beim Adjektiv cf. Ros 1992). Diese lässt sich für das Suffix -ig attestieren, cf.: für Verben ein ?surfiges Meer/Das Wasser ist ??surfig/Er bewegt sich ??surfig (> surfen), ein ?downloadiges Portal/Das Portal ist?downloadig; Ute surft ?? downloadig (> downloaden); eine ?trackige Internetsuche/Die Person ist ??trackig/Google sucht ??trackig nach Personen (> tracken > engl. to track, verfolgen'); sowie für Substantive: Das ?kalenderige Bild/Das Bild ist ziemlich ?kalenderig/Er malt??kalendrig/Der ?mozartige Gast/Hans ist ?mozartig/Lisa spielt die Sonate ??mozartig; Der ?tischige Stuhl (,Stuhl wie ein Tisch')/Dieser Stuhl ist breit, ja richtig ?tischig/Dieser Stuhl wirkt??tischig. Auch wenn diese Bildungen synchron möglicherweise noch bildbar und in bestimmten Aktualisierungskontexten interpretierbar sind, zeichnet sich doch die Tendenz zur Ersetzung durch andere Kodierungsformen ab, so durch das Suffix -bar bei deverbaler Basis (cf. surfbar/chillbar/trackbar) und durch v. a. mündlich vorkommende Suffixoide bei substantivischer Basis (cf. kalendermäßig/mozartmäßig/tischartig im Sinne Fandrychs (2011). Insbesondere scheint das Suffix -ig auf Vergleiche implizierende Kontexte eingeschränkt (sog. imitative Semantik) und für Simplicia als Basen zunehmend pejorativ zu sein, d. h. semantisch wie pragmatisch restringiert. 
Während bei den Substantiven keinerlei Suffixoide wirklich die Kraft zu haben scheinen, als zukünftige Suffixe zu fungieren, ist dies bei den Adjektiven zu relativieren. Wie Fandrych (2011) gezeigt hat, geht es gar nicht um ein einzelnes adjektivisches Affix, sondern vielmehr entwickelt sich gerade ein adjektivisches „Kompositionsmuster”, das Rektionskomposita bildet, unabhängig davon, ob das zugrundeliegende Adjektiv (als Zweitglied) überhaupt eine Rektionspotenz hat, wie zum Beispiel bei arbeitsfähig, arbeitswillig, arbeitswütig, arbeitsfreudig usw. (cf. Fandrych 2011: 137), wobei die Wortstrukturen durchaus unterschiedlich interpretiert werden können, sich aber doch jeweils Tendenzen zu Reihenbildung zeigen. Bei den Adjektiven zeigt sich also eine Konstruktion, die eine bestimmte Interpretation beinhaltet. Es sind aber keine speziellen Formen und somit vermutlich auch keine Suffixe der Zukunft, sondern wie Fandrych sie nennt „lexematische Junktionsglieder“ (2011: 152). Die Tendenz zur Zunahme der Konversion zeigt sich hier weniger deutlich im Vergleich zu Nominalisierungen.

Bei den Verben hat das Deutsche schon lange kein wortbildendes Suffix mehr. Das letzte war -ier-, was sich für native Basen nicht durchsetzen konnte und bei den nicht-nativen heutzutage nicht mehr produktiv genutzt wird. Bei den Adverbien hat es schon lange keine eindeutigen Suffixe mehr (zur diachronen Entwicklung cf. Pounder 2001) gegeben, wohl aber finden sich hier vereinzelt Umdeutungen wie donnerstag statt donnerstags.

Damit lässt sich zusammenfassen: Die Entwicklung und diachrone Zunahme der Suffixe im Bereich der Substantive und Adjektive ist ein bekanntes sprachhistorisches Faktum des Deutschen. Ererbte, unproduktive Derivationsbildungen des Gegenwartsdeutschen (wie - de, - $t$ oder -nis im substantivischen Bereich sowie -sam, -lich oder -lings) sind ein Abbild dieser einstigen Produktivität und zeigen gleichzeitig, dass suffixale Muster ,absterben“, d. h. durch andere Kodierungsformen ersetzt werden können. Insbesondere der Bereich der Nominalisierungen zeigt hier Tendenzen zu einem Kodierungswechsel hin zur (syntaktischen) Konversion, d. h. einem inhärent syntaktischen Prozess, welcher für die Verben seit jeher ungleich häufiger konstatiert wurde. Die morphosyntaktische „Schnittstelle“ zwischen Nomina und Verben stellen die deverbalen Nominalisierungen dar, deren prominenteste Entwicklung von der Derivation zur Konversion in der Aufgabe der -ung-Nominalisierungen bei gleichzeitiger Produktivitätserhöhung des substantivischen Infinitivs seit mhd. Zeit besteht. Mit den hier skizzierten Entwicklungstendenzen ist nicht ausgesagt, dass diese Entwicklung auf jeden Fall eintreten muss (wie es die Teleologie nahelegen würde). Stattdessen handelt es sich vor dem Hintergrund synchroner wie diachroner Befunde um kein zu verabsolutierendes, aber wahrscheinliches Szenario. Im vorliegenden Beitrag wurde dafür argumentiert, dass Wortbildungssuffixe zwar Wörter bilde(te)n, aber die produktiv gebildeten Wörter im Gegenwartsdeutschen dennoch nicht (nur) als Teile eines immer größer werdenden Lexikons fungieren, sondern regelbasierte Einheiten sind, deren Bildungsprinzipien stets dynamischen Gesetzmäßigkeiten unterliegen. Diachron betrachtet nimmt also einerseits der Bestand der dokumentierbaren Suffixe bis zum Gegenwartsdeutschen zu, andererseits kann deren jeweilige Produktivität musterbildend schwinden. Nach traditioneller Auffassung machen sie dadurch Platz für neue Suffixe. Im vorliegenden Beitrag wurde jedoch gezeigt, dass auch andere morphosyntaktische Prozesse (wie bspw. die syntaktische Konversion) suffixale Muster ablösen können, so dass man möglicherweise von einer zunehmenden „Syntaktisierung“ sprechen könnte. Sprachtypologisch erklärt die Theorie der Syntaktisierung möglicherweise auch, warum bzw. wie aus einer derivationsreichen eine derivati- 
onsarme Sprache werden kann, während der umgekehrte Prozess diachron (Komposition > Derivation) bereits vielfach beschrieben wurde. Da im konkreten Fall jeweils eigenständige Wörter erhalten bleiben und nur in ihrer morphologischen Struktur verändert werden, handelt es sich unter dem Aspekt des morphologischen Sprachwandels um einen morphosyntaktischen Kodierungs-Zyklus, der innerhalb der Dimensionen von Markiertheit und Unmarkiertheit verortet werden kann.

\section{Literatur}

Alexiadou, Artemis/Iordachioaia, Gianina/Schäfer, Florian (2011): "Scaling the variation in Romance and Germanic nominalizations". In: Sleeman, Petra/Perridon, Harry (eds.): The Noun Phrase in Romance and Germanic. Amsterdam, Benjamins: 25-40.

Altmann, Hans/Kemmerling, Silke (2000): Wortbildung fürs Examen. Wiesbaden: VS Verlag für Sozialwissenschaften.

Aronoff, Mark (1976): Word-formation in Generative Grammar. Cambridge: MIT Press.

Baayen, R. Harald/Renouf, Antoinette (1996): "Chronicling The Times: Productive lexical innovations in an English newspaper”. Language 72: 69-96.

Bakema, Peter/Geeraerts, Dirk (2004): "Diminution and augmentation". In: Booij, Lehmann et al. (eds.): Morphologie/Morphology 1: 1045-1052. (= Handbücher zur Sprach- und Kommunikationswissenschaft /Handbooks of Linguistics and Communication Science 17/1).

Bauer, Laurie (2004): Morphological productivity. Digital print ed. Cambridge: Cambridge University Press.

Bech, Gunnar (1955/1983): Studien über das deutsche Verbum infinitum. 2., unveränd. Aufl., mit einem Vorwort von Cathrine Fabricius-Hansen. Tübingen: Niemeyer.

Becker, Thomas (1990): Analogie und morphologische Theorie. München: Fink.

Bredel, Ursula (2006): „Die Herausbildung des syntaktischen Prinzips“. In: Bredel, Ursula/Günther, Hartmut (eds.): Orthographietheorie und Rechtschreibunterricht. Tübingen, Niemeyer: 139-164.

Buchmann, Franziska (2015): Die Wortzeichen im Deutschen. Heidelberg: Winter.

Bücking, Sebastian (2012): „Müdigkeit und Müde-Sein“. Linguistische Berichte 232: 361-397.

Bülow, Lars/Herz, Matthias (2014): „Semantische Kämpfe um Wissenschaftlichkeit und Ideologie: Gender Studies, ihre Gegner/innen und die Konsequenzen für den Sprachgebrauch und das Sprachsystem“. Linguistische Berichte 240: 471-516.

Chan, Sze-Mun (2005): Genusintegration. Eine systematische Untersuchung zur Genuszuweisung englischer Entlehnungen in der deutschen Sprache. München: Iudicium.

Demske, Ulrike (2000): „Zur Geschichte der ung-Nominalisierung im Deutschen“. PBB 122/3: $365-411$.

Draeger, Kerstin (1996): Die semantische Leistung der suffixalen Wortbildungsmorpheme der Substantive in der deutschen Gegenwartssprache. Aachen: Shaker.

Eichinger, Ludwig (2000): Deutsche Wortbildung. Eine Einführung. Tübingen: Narr.

Eisenberg, Peter (2002): „Morphologie und Distribution - Zur Morphosyntax von Adjektiv und Adverb im Deutschen“. In: Schmöe, Friederike (ed.): Das Adverb. Zentrum und Peripherie einer Wortklasse. Wien, Edition Präsens: 61-76. 
Eisenberg, Peter/Sayatz, Ulrike (2005): "Left of Number. Animacy and Plurality in German Nouns”. In: Gunkel, Zifonun et al. (eds.): Explorations in Nominal Inflection. Berlin, de Gruyter: 97-120.

Eisenberg, Peter (2012): Das Fremdwort im Deutschen. 2. Auflage. Berlin: de Gruyter.

Eisenberg, Peter (2013a): „Anglizismen im Deutschen“. In: Deutsche Akademie für Sprache und Dichtung (ed.): Reichtum und Armut der deutschen Sprache. Erster Bericht zur Lage der deutschen Sprache. Berlin, de Gruyter: 57-119.

Eisenberg, Peter (2013b): Grundriss der deutschen Grammatik. Das Wort. 4. Auflage. Stuttgart: Metzler.

Eisenberg, Peter (2013c): Grundriss der deutschen Grammatik. Der Satz. 4. Auflage. Stuttgart: Metzler.

Fandrych, Christian (2011): „Wie geht es eigentlich den ,Halbsuffixen“”?“ In: Deutsche Sprache 2/2011: 137-153.

Fleischer, Wolfgang (1976): Wortbildung der deutschen Gegenwartssprache. Leipzig: VEB Bibliographisches Institut Leipzig.

Fleischer, Wolfgang/Barz, Irmhild (2012): Wortbildung der deutschen Gegenwartssprache. 4. Auflage. Berlin: de Gruyter.

Fuhrhop, Nanna (1998a): Grenzfälle morphologischer Einheiten. Tübingen: Stauffenburg.

Fuhrhop, Nanna (1998b): “A derivational analysis of foreign stems". ZAS Working Papers in Linguistics 13: 38-44.

Fuhrhop, Nanna/Teuber, Oliver (2000): „Das Partizip 1 als adjektivischer Infinitiv“. In: Bittner, Köpcke et al. (eds.): Angemessene Strukturen: Systemorganisation in Phonologie, Morphologie und Syntax. Hildesheim, Olms: 173-190.

Gaeta, Livio (2010): „Polysynthese, Multifunktionalität und die denominalen Adjektive im Deutschen“. In: Bittner, Dagmar/Gaeta, Livio (eds.): Kodierungstechniken im Wandel. Berlin, de Gruyter: 99-121.

Gaeta, Livio/Ricca, Davide (2015): "Restrictions in word-formation". In: Müller, Ohnheiser et al. (eds.): Wortbildung/Word formation. Bd. 2. Berlin, de Gruyter: 859-875. (= Handbücher zur Sprach- und Kommunikationswissenschaft/Handbooks of Linguistics and Communication Science 40/2).

Höhle, Tilman (1982): „Über Komposition und Derivation: zur Konstituentenstruktur von Wortbildungsprodukten im Deutschen“. Zeitschrift für Sprachwissenschaft 1: 76-112.

Kloocke, Hella (1974): Der Gebrauch des substantivierten Infinitivs im Mittelhochdeutschen. Göppingen: Kümmerle.

Köpcke, Klaus-Michael (1995): „Die Klassifikation der schwachen Maskulina in der deutschen Gegenwartssprache“. Zeitschrift für Sprachwissenschaft 14: 159-180.

Lieb, Hans Heinrich (1983): Integrative Linguistics. Vol. I: General Outlines. Amsterdam: Benjamins.

Lübbe, Anja (2013): „Das regulär prädikative Partizip I“. Deutsche Sprache 2/13: 97-114.

Lüdeling, Anke (2001): On Particle Verbs and Similar Constructions in German. Stanford: CSLI.

Maienborn, Claudia (2007): „Das Zustandspassiv“. Zeitschrift für Germanistische Linguistik 35: 83-114.

Moser, Stefan (1993): Substantivische Affixbildung im Frühneuhochdeutschen. Diss., Universität Würzburg. 
Nübling, Damaris (2013): Historische Sprachwissenschaft des Deutschen. 4. Auflage. Tübingen: Narr.

Olsen, Susan (1996): „Partikelverben im deutsch-englischen Vergleich“. In: Lang, Ewald/Zifonun, Gisela (eds.): Deutsch - typologisch. Berlin, de Gruyter: 261-288.

O'Reilly, Tim (2007): "What is Web 2.0: design patterns and business models for the next generation of software". Communications \& Strategies 65: 17-21.

Pounder, Amanda (2001): "Adverb-marking in German and English. System and standardization”. Diachronica XVIII/2: 301-358.

Rapp, Irene (1996): „Zustand? Passiv? Überlegungen zum sogenannten Zustandspassiv“. Zeitschrift für Sprachwissenschaft 15/2: 231-265.

Reis, Marga (1983): „Gegen die Kompositionstheorie der Affigierung“. Zeitschrift für Sprachwissenschaft 2: 110-131.

Ros, Gisela (1992): Suffixale Wortbildungsmorpheme. Untersuchungen zu ihrer semantischen Leistung am Beiwort der deutschen Gegenwartssprache. Stuttgart: Akademischer Verlag. (= Stuttgarter Arbeiten zur Germanistik 258).

Schäfer, Florian (2012): "Naturally atomic er-nominalizations". Recherches linguistiques de Vincennes 40: 149-174.

Scherer, Carmen (2005): Wortbildungswandel und Produktivität. Eine empirische Studie zur nominalen -er-Derivation im Deutschen. Tübingen: Niemeyer.

Schneider-Wiejowski, Karina (2011): Produktivität in der deutschen Derivationsmorphologie. http://pub.uni-bielefeld.de/publication/2473126 [15.12.2014].

Scott, Alan (2009): „Denominales -er: Ein Suffix lebt wieder auf“. Zeitschrift für germanistische Linguistik 37/2: 221-235.

Werner, Martina (2010): „Substantivierter Infinitiv statt Derivation“. In: Bittner, Dagmar/Gaeta, Livio (eds.): Kodierungstechniken im Wandel. Berlin, de Gruyter: 159-178.

Werner, Martina (2012): Genus, Derivation und Quantifikation. Zur Funktion der Suffigierung und verwandter Phänomene im Deutschen. Berlin/Boston: de Gruyter. (Studia Linguistica Germanica 114).

Wilmanns, Wilhelm (1896): Deutsche Grammatik. Band 2: Wortbildung. Strassburg: Trübner. 